\title{
Thrower's fracture of the humerus with radial nerve palsy: an unfamiliar softball injury
}

\section{P Curtin, C Taylor, J Rice}

Br J Sports Med 2005;39:e40 (http://www.bjsportmed.com/cgi/content/full/39/11/e40). doi: 10.1136/bjsm.2004.016345

\begin{abstract}
A fracture of the normal humerus in a healthy young adult most commonly results from significant direct trauma. Throwing sports have become increasingly popular outside of North America and bring with them a novel injury mechanism for clinicians. A 21 year old woman sustained a "thrower's fracture" of the distal humerus and radial nerve palsy while throwing a softball. She was treated by internal fixation. Her fracture united, and radial nerve neurapraxia resolved after 8 weeks. Clinicians should be aware of this entity so that prodromal symptoms can be recognised early and thrower's fractures are not investigated unnecessarily.
\end{abstract}

$\mathrm{H}$ umeral fractures in patients $<50$ years of age with normal bone usually only occur after direct trauma, whereas in the elderly they often occur after simple falls and indirect injuries. ${ }^{1}$ Humeral fractures due to extreme muscular forces occurring while throwing a stone, snowball, javelin, hand grenade, baseball, or softball are rare but described mechanisms in the literature. ${ }^{2-10}$ We recently encountered a fracture of the distal humerus with concomitant radial nerve palsy in a healthy young adult that resulted from throwing a softball. This injury, eponymously known as a Holstein-Lewis fracture when it occurs with a radial nerve neurapraxia, ${ }^{11}$ has not been reported by this mechanism in the British literature.

Awareness of this injury mechanism is relevant for all clinicians, as prodromal pain occurring in the throwing arms of softball or baseball players may indicate a stress fracture. Furthermore, as we have learned from this case, further imaging to exclude a pathological fracture is unnecessary.

\section{CASE REPORT}

A 21 year old right handed woman developed pain and swelling in her right upper arm immediately following an "overarm throw" while playing in the "outfield" in a softball game. She presented at the emergency department the following day when she developed associated weakness in her right wrist. She had been playing softball 2-3 times weekly for 8 months, and had complained of pain on the medial aspect of her left elbow for 2 months, which had been treated as medial epicondylitis. On examination, her upper arm was swollen and tender, with an angular deformity, and she had a radial nerve palsy, with no active finger or wrist extension and anaesthesia over the first web space dorsally. An $x$ ray showed a spiral fracture of the junction of middle and distal thirds of the shaft of her right humerus (fig 1).

Dynamic splinting was initiated immediately postoperatively to facilitate radial nerve recovery. Two months following her injury, her radial nerve neurapraxia had resolved. Wrist and finger extension were grade $4 / 5$ and radial nerve sensation had returned to normal. Range of motion of her elbow and wrist were $70 \%$ of normal. Both clinically and radiologically (fig 3 ), her fracture was uniting.

After admission, we performed investigations to exclude a pathological bone lesion. A bone scan and magnetic resonance imagingof her upper limb did not show any additional abnormality (fig 2). Internal fixation of her fracture was performed through a posterior triceps splitting approach. The radial nerve was not found to be related to the fracture site at the time of fixation.

\section{DISCUSSION}

Fracture of the humerus from an overarm throw occurs rarely, especially considering the popularity of throwing
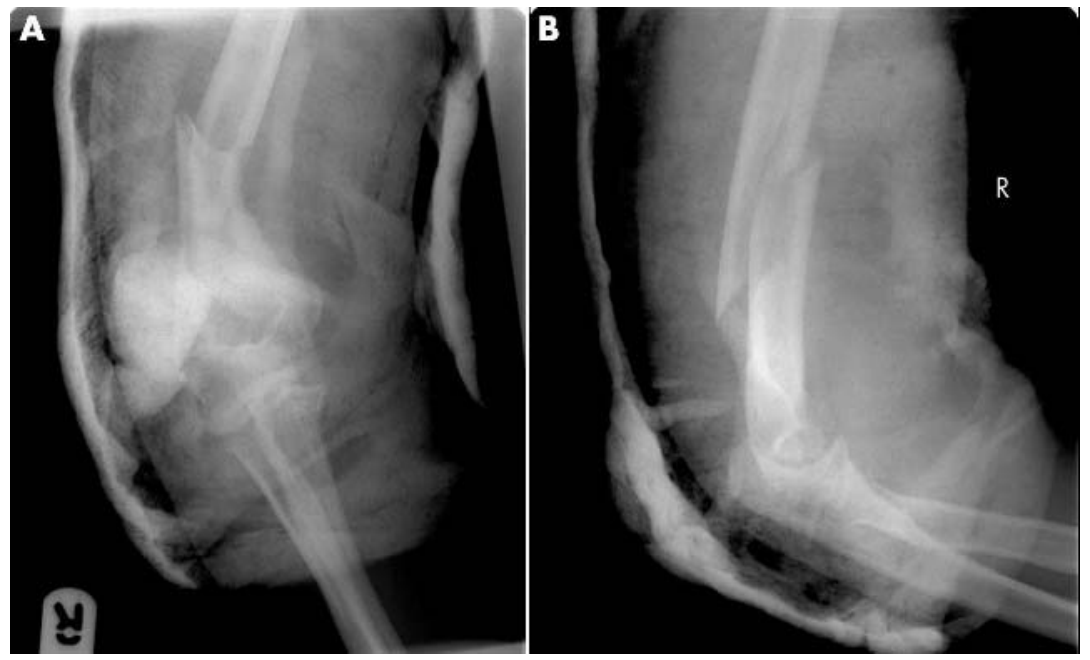

Figure 1 AP and lateral radiographs of the elbow and distal humerus showing a spiral fracture of the junction of middle and distal thirds of the humerus in a coaptation splint or $U$ slab. 

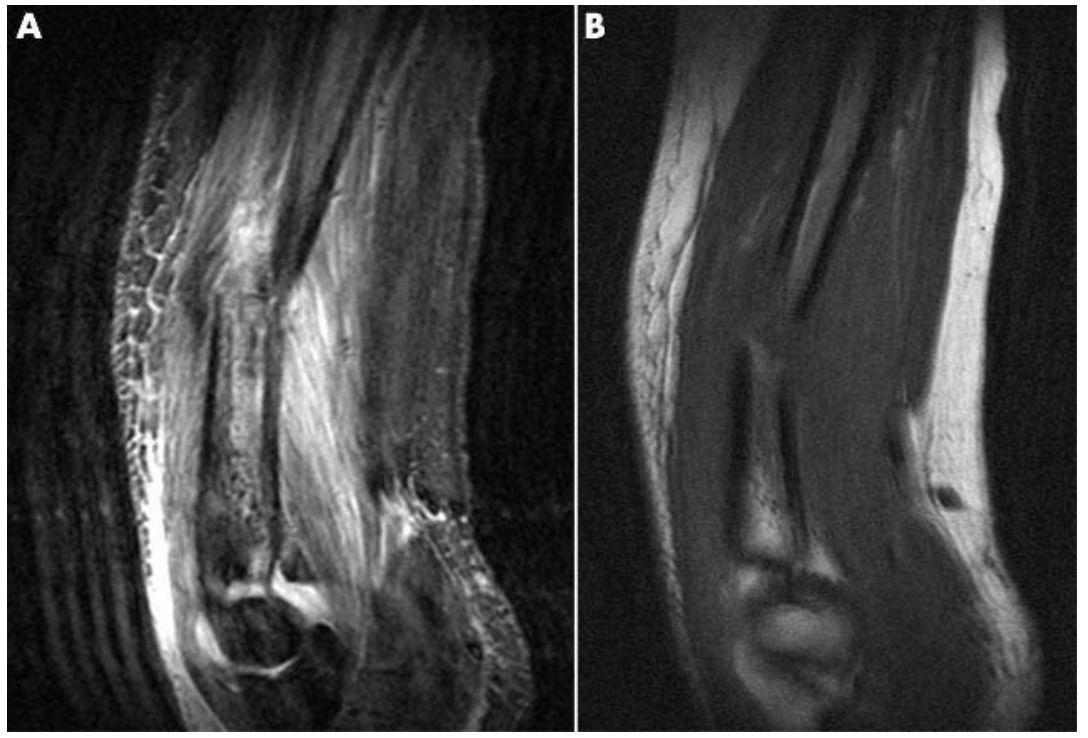

Figure 2 Sagittal $\mathrm{T} 1$ and T2 magnetic resonance imaging of the fracture show no evidence of a pathological bone or soft tissue lesion.

sports in North America and the small numbers reported in the literature. ${ }^{1-10}$ Torsional forces or torque created around the long axis of the arm in the final phase of throwing typically give rise to spiral fractures in the middle to distal humerus. ${ }^{23}$ Humeral torque is proportional to the mass of the object being thrown, and a standard softballand is heavier than a baseball, weighing in at $193 \mathrm{~g}$.

Thrower's fractures are more common in recreational throwers because of unsynchronised muscular activity and uncoordinated throwing styles. ${ }^{2-10}$ Amateur players tend to activate all the rotator cuff muscles as well as the biceps for power in an unsynchronised inappropriate manner, whereas professionals selectively activate rotator cuff muscles during the throwing motion in a well conditioned and well synchronised fashion. ${ }^{3}$

A succession of muscular forces is transmitted through the humerus during the three phases of throwing. During the first, cocking phase, the humerus is abducted and brought into extreme external rotation by the deltoid and rotator cuff muscles respectively. During the second phase, acceleration, the arm is brought into flexion and internal rotation by the pectoralis major, latissimus dorsi, teres major, coracobrachialis and subscapularis. In the final deceleration phase, the largest amount of muscle activity occurs, when the rotator cuff and deltoid decelerate the arm and the elbow decelerates from extreme extension to abrupt flexion by the action of the biceps. At the point when external rotation is transferred to internal rotation and when flexion of the elbow changes to extension, the torsional force is at its maximum and this is the point where fracture occurs. ${ }^{3}$

Prodromal pain in the shoulder, elbow or eventual fracture site has been reported in up to $50 \%$ of cases. ${ }^{2}$ In this case presented, the fracture occurred following an extreme effort throw and there was a history of prodromal pain around the eventual fracture site which went unrecognised. A radial nerve neurapraxia occurred because the nerve is tethered as it pierces the lateral intermuscular septum and does not yield when the distal humeral fragment displaces. ${ }^{11}$ We performed
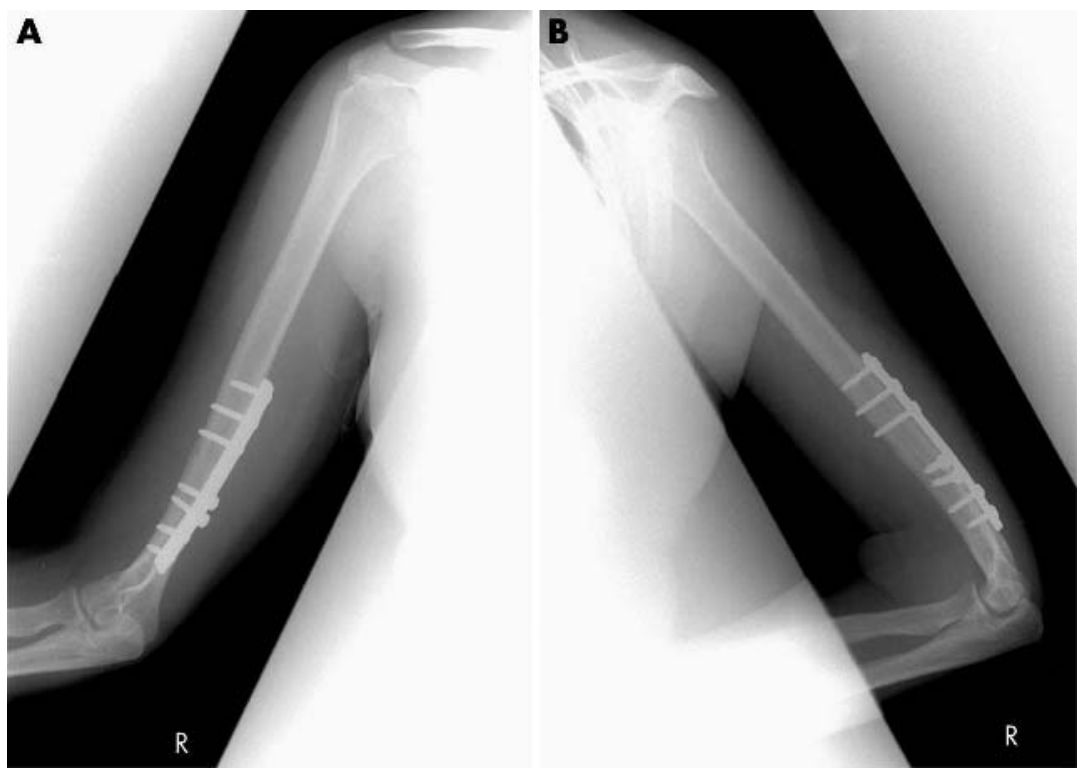

Figure 3 Follow up AP and lateral radiographs of the humerus at 8 weeks following open reduction and internal fixation showing progress to radiological union of the fracture. 


\section{What is already known on this topic}

- The mechanism of this injury is reported sporadically in the literature but is nevertheless not widely recognised.

- The fascinating biomechanics of the injury have been reported and the mechanism of radial nerve neurapraxia in humeral fractures is known.

\section{What this study adds}

- Although case reports are a less than perfect source of evidence, this short report alerts the clinician to a rare injury that often occurs with prodromal symptoms.

- A brief review of the biomechanics and the mechanism of radial nerve neurapraxia helps the clinician understand the injury and avoid unnecessary investigations.

further imaging to exclude a pathological fracture of the humerus but subsequently found this to have been unnecessary following review of the literature. Fractures of the distal humerus can be treated conservatively with excellent results, ${ }^{2-8}$ even when there is a concomitant radial nerve palsy. ${ }^{27}$ In this young woman we performed internal fixation to avoid the possibility of a malunion of the distal humerus which would have resulted in an unsightly angular deformity, and the patient went on to make an excellent recovery.
Authors' affiliations

P Curtin, C Taylor, J Rice, Adelaide and Meath Hospital, Republic of Ireland

Competing interests: none

The patient described in this article consented to the publication of her details

Correspondence to: Dr P Curtin, Adelaide and Meath Hospital, Trauma and Orthopaedics, Dublin, Ireland; pauldcurtin@hotmail.com

Received 10 February 2005

In revised form 19 March 2005

Accepted 21 March 2005

\section{REFERENCES}

1 Tytherleigh-Strong $\mathbf{G}$, Walls $N$, et al. The epidemiology of humeral shaft fractures. J Bone Joint Surg (Br) 1998;80-B:249-53.

2 Ogawa K, Yoshida A. Throwing fracture of the humeral shaft. An analysis of 90 patients. Am J Sports Med 1998;26:242-6.

3 Sabick MB, Torry MR, Kim YK, et al. Humeral torque in professional baseball pitchers. Am J Sports Med 2004;32:892-8.

4 Callaghan EB, Bennett DL, El-Khoury GY, et al. Ball-thrower's fracture of the humerus. Skeletal Radiol 2004;33:355-8.

5 DiCicco JD, Mehlman CT, Urse JS. Fracture of the shaft of the humerus secondary to muscular violence. J Orthop Trauma 1993;7:90-3.

6 Kuschner SH, Lane CS. Recurrent fracture of the humerus in a softball player. Am J Orthop 1999;28:654-6.

7 Kaplan H, Kiral A, Kuskucu M, et al. Report of eight cases of humeral fracture following the throwing of hand grenades. Arch Orthop Trauma Surg 1998;117:50-2.

8 Weseley MS, Barenfeld PA. Ball throwers' fracture of the humerus. Six case reports. Clin Orthop Relat Res 1969;64:153-6.

9 Evans PA, Farnell RD, Moalypour S, et al. Thrower's fracture: a comparison of two presentations of a rare fracture. J Accid Emerg Med 1995;12:222-4.

10 Bontempo E, Trager SL. Ball thrower's fracture of the humerus associated with radial nerve palsy. Orthopedics 1996;19:537-40.

11 Holstein A, Lewis GB. Fractures of the humerus with radial-nerve paralysis. $J$ Bone Joint Surg (Am) 1963;45:1382-8. 